\title{
Microstructure of Dental Amalgam
}

\author{
FERNE C. ALLAN, KAMAL ASGAR, and F. A. PEYTON
}

University of Michigan, School of Dentistry, Ann Arbor, Michigan

Numerous studies have been done in the past to identify the phases present in dental amalgam. Both metallographic and X-ray diffraction techniques have been used. ${ }^{1-6}$ Most researchers now presume that dental amalgam consists mainly of three phases. The original particle is an alloy of $\mathrm{Ag}, \mathrm{Sn}$, $\mathrm{Cu}$, and sometimes $\mathrm{Zn}$; the major portion is the compound $\mathrm{Ag}_{3} \mathrm{Sn}$, which is often referred to as $\gamma$. After amalgamation, a portion of the original particle is left; in addition, an $\mathrm{Ag}_{2} \mathrm{Hg}_{3}\left(\gamma_{1}\right)$ phase and a $\mathrm{Sn}-\mathrm{Hg}\left(\gamma_{2}\right)$ phase form. Schmitt ${ }^{1}$ and Wing $^{2}$ have described the $\gamma$ and $\gamma_{1}$ phases in the microstructure. However, the $\gamma_{2}$ phase has not been identified metallographically, except by pointing out voids where apparently a phase was present.

Other phases have been proposed, but their existence has not been established. Although $\mathrm{Cu}$ may be present in the alloy in a proportion as high as 6 per cent, this element has not been identified in the structure. Wing ${ }^{2}$ and Crowell ${ }^{3}$ both have postulated that a dark or gray etch-resistant area which appears in the alloy particles may be a $\mathrm{Cu}$-Sn phase.

Since the polishing methods which have been used in the past are not consistent, we developed a standard procedure for metallographic polishing which does not erode the $\gamma_{2}$ areas. In addition, an etching solution was developed which reveals all the phases in amalgam without etching out the more reactive phases.

After examination of various microstructures, a typical microstructure was defined. Then the three major phases were identified in the structure. Further work in this area will depend on a more positive identification of the phases and of the elements present.

Presented before the Dental Materials Group of the IADR at Los Angeles, California, March, 1964.

This study was supported by a grant from the Office of Naval Research, Contract No. NR-180-360, to the University of Michigan, School of Dentistry.

Received for publication July 21, 1964.
The electron probe microanalyzer has been developed as a practical research instrument for the analysis of small areas in situ. Therefore, several exploratory tests were run on this instrument to test its applicability in the study of dental amalgam.

\section{Materials and Methods}

PReparation AND POLIShING OF THE SPECIMENS.-No special techniques or materials are necessary for the metallographic polishing of dental amalgam. However, because of the low melting point of the alloy, care must be used to maintain a light pressure and to avoid overheating the sample. The samples were representative of amalgams used in dentistry. Normal trituration methods and condensation procedures were used. The triturated mass was condensed directly into cavities prepared in acrylic blocks. These samples were allowed to set for a week before being polished.

Standard metallographic procedure was followed. ${ }^{7,8}$ Rough polishing was done on successively finer grades of emery paper $(3,2,1,1 / 0,2 / 0,3 / 0,4 / 0)$. When the $4 / 0$ emery paper was used, less time was needed on the first wheel and the $\mathrm{Sn}-\mathrm{Hg}$ phase showed better retention.

Four laps were used in the following order: broadcloth with $15 \mu$ polishing alumina (powder) suspension; broadcloth with $5 \mu$ polishing alumina (No. 1);* microcloth $\dagger$ with $0.3 \mu$ polishing alumina (No. 2 ); $\ddagger$ and microcloth with chromium oxide. \$ The final polish with chromium oxide was done lightly for 5-10 seconds. The samples were washed with soap, dried in an air blast, and stored in a desiccator for the minimum time possible before etching.

* AB Polishing Alumina No. 1, Buehler, Ltd., Evarston, Ill $\uparrow$ AB Microcloth, Buehler, Ltd., Evanston, Ill.

$\ddagger$ AB Alpha Polishing Alumina No. 2, Buehler, Ltd., Evan ston, IIl.

$\S \mathrm{C}-\mathrm{RO}$, Burrell Corp., Pittsburgh, Pa. 
ETCHING OF THE SPECIMENS.-The microstructure of dental amalgam is complex. In addition, the chemical reactivities of the phases vary greatly. ${ }^{1}$ Although many etching solutions have been proposed in the literature, each has a shortcoming which limits its use.

Both dental ${ }^{1-4}$ and metallographic literatures $^{8-10}$ were surveyed to find an etch suitable for amalgam. Many of these etchants, plus experimental etchants devised in our laboratory, were tried. The etch which was developed and considered most suitable consists of:

\begin{tabular}{llc}
\multicolumn{1}{c}{ Solution A } & Solution B & "Hypo" Rinse \\
$4 \mathrm{gm} . \mathrm{K}_{2} \mathrm{Cr}_{2} \mathrm{O}_{7}$ & $4 \mathrm{gm} . \mathrm{I}$ & $\mathrm{Na}_{2} \mathrm{~S}_{2} \mathrm{O}_{3} \cdot 5 \mathrm{H}_{2} \mathrm{O}$ \\
$1 \mathrm{gm} . \mathrm{KI}$ & $96 \mathrm{ml}$. ethyl & Dissolved in \\
$100 \mathrm{ml} \mathrm{H}_{2} \mathrm{O}$ & alcohol & water
\end{tabular}

The sample was swabbed with Solution A for 20-40 seconds and rinsed with water. Then it was lightly swabbed with Solution B for 5-15 seconds, followed with a "hypo" swab-rinse, washed with water, and dried.

The "hypo" rinse functions to remove any silver salts which form. This solution should be replaced frequently to assure uniform results. Solution A functions mainly as a pre-etch and controls the degree of phase contrast obtained. The resultant etch is affected to the greatest extent by the contact time and pressure during swabbing of Solution B. This solution brings out detail and depth of the unreacted original particle and the $\mathrm{Ag}_{2} \mathrm{Hg}_{3}$ phase. The microstructure of various amalgam samples was studied, and representative areas were selected for analysis with the microprobe.

USE OF THE MICROPROBE. *-The phases present in dental amalgam are, by a metallographic comparison, quite small in size. The grain size of $\mathrm{Ag}_{2} \mathrm{Hg}_{3}$ varies from 1-10 $\mu$, and the original particles are approximately $50 \mu$. The $\gamma_{2}$ phase is about $20 \mu$ in size, and, in addition, is quite irregular. Therefore, variations in composition of these areas are almost indistinguishable and cannot be identified microscopically.

In order to gain an idea of what elements are present in these phases, a few typical areas were selected for analysis by the electron probe microanalyzer.

This instrument has been gaining wide

* Norelco AMR/3 Electron Probe Microanalyzer, Philips Electronic Instruments, Mt. Vernon, N.Y. acceptance in the past few years since it can chemically analyze small areas (approximately $1 \mu$ ) in situ. Essentially, the area being analyzed is made the target of an $\mathrm{X}$-ray tube. When the area is bombarded by a beam of electrons, the atoms of each element are excited to the level of emitting their characteristic X-rays. Spectrometric analysis then permits each element present to be determined, since every element has its own characteristic wave length. This determination is on a chemical basis, not on the more commonly known basis of crystal structure, as in X-ray diffraction. By comparison with standards, the per cent of each element present can be determined.

The probe adapts the optic system of the electron microscope in order to permit focusing of the beam of bombarding electrons on such a small area. The probe also has a built-in microscope for aligning and viewing of the samples. ${ }^{11}$

Areas selected to be analyzed were the light gray etch-resistant areas and the dark gray areas which appear in aged amalgam. The samples were polished in a normal procedure, and the areas to be analyzed were marked with a diamond indenter and photographed at $900 \times$. $\dagger$ The probe-beam diameter was focused to include the entire phase, and the spectrum of elements was scanned. Once the range of elements present was established, scans were run for this range. As a check, a normal area on each specimen was also analyzed. After analysis, the exact area covered by the beam was outlined on the photograph taken previously.

The $\gamma_{2}$ areas were also analyzed. The area was located by the viewing system of the probe. Scans were first made for the entire spectrum and then for specific elements at various points within the phase.

Because the sample is used as an electron target, the possibility exists that the amalgam might be overheated and the structure changed. The samples were flash-coated with carbon to increase electrical conductivity. The surfaces were examined after analysis and showed no evidence of overheating.

t Since the viewing system of the probe is $400 \times$ magnification, these photomicrographs were taken in a metallographic microscope.

$\ddagger$ The strongest lines for the elements of interest were in the range of $34^{\circ}-50^{\circ}$. 


\section{Results and Discussion}

Polishing AND ETCHING.-The surface obtained by using the recommended metallographic procedure is shown in Figure 1. All the phases are retained, and the voids are easily distinguishable. When polishing is continued to the point that the original particles show in relief, the sample has been overpolished and some of the $\gamma_{2}$ areas will appear as voids.

Since the composition of different alloys varies, an etch is needed with which the rate and degree of etching can be controlled. Also the etch should reveal the original particle in the amalgam and should be usable on both alloy and amalgam. It must distinguish between the phases and give a good contrast. In addition, all phases should be retained.

The ferric chloride etch ${ }^{1}$ destroys the $\gamma_{2}$ phase, does not bring out detail in the $\gamma_{1}$ phase, and produces artifacts on the structure (Fig. 2). It is also inadequate for etching alloy particles. The cyanide etch ${ }^{2}$ does not give good phase contrast in the amalgam (Fig. 3), although it is suitable for revealing the Widmanstatten structure in the particles. This structure helps to identify the original unreacted particles in the amalgam. Although the unreacted particles and the matrix are well shown in the amalgam, the $\gamma_{2}$ portion of the matrix cannot be identified. The nitric acid etch ${ }^{4}$ shows the $\gamma_{1}$ grain boundaries well but etches out completely the $\gamma_{2}$ phase and does not give detail in the unreacted particle (Fig. 4). With all these etches, voids cannot be distinguished and are confused with the $\gamma_{2}$ phase.

Figure 5 is representative of the results obtainable with the iodine etch. The three phases can be distinguished at one time, with detail being shown in the original unreacted particles and $\gamma_{1}$. The unreacted particles appear gray, with the Widmanstatten structure (Fig. 6) appearing dark (brown or shades of blue and green). The $\mathrm{Ag}_{2} \mathrm{Hg}_{3}\left(\gamma_{1}\right)$ phase appears as a matrix of light gray, small, irregular grains. The $\gamma_{2}$ areas etch in dark shades of purples, blues, and greens and are distinguishable from the voids. When viewed in the microscope, the voids show as black, out-of-focus areas; however, this difference does not reproduce well in the conventional black and white photomicrographs. Since the reaction of the $\gamma_{2}$ areas to the etch varied, these areas were studied further by probe.

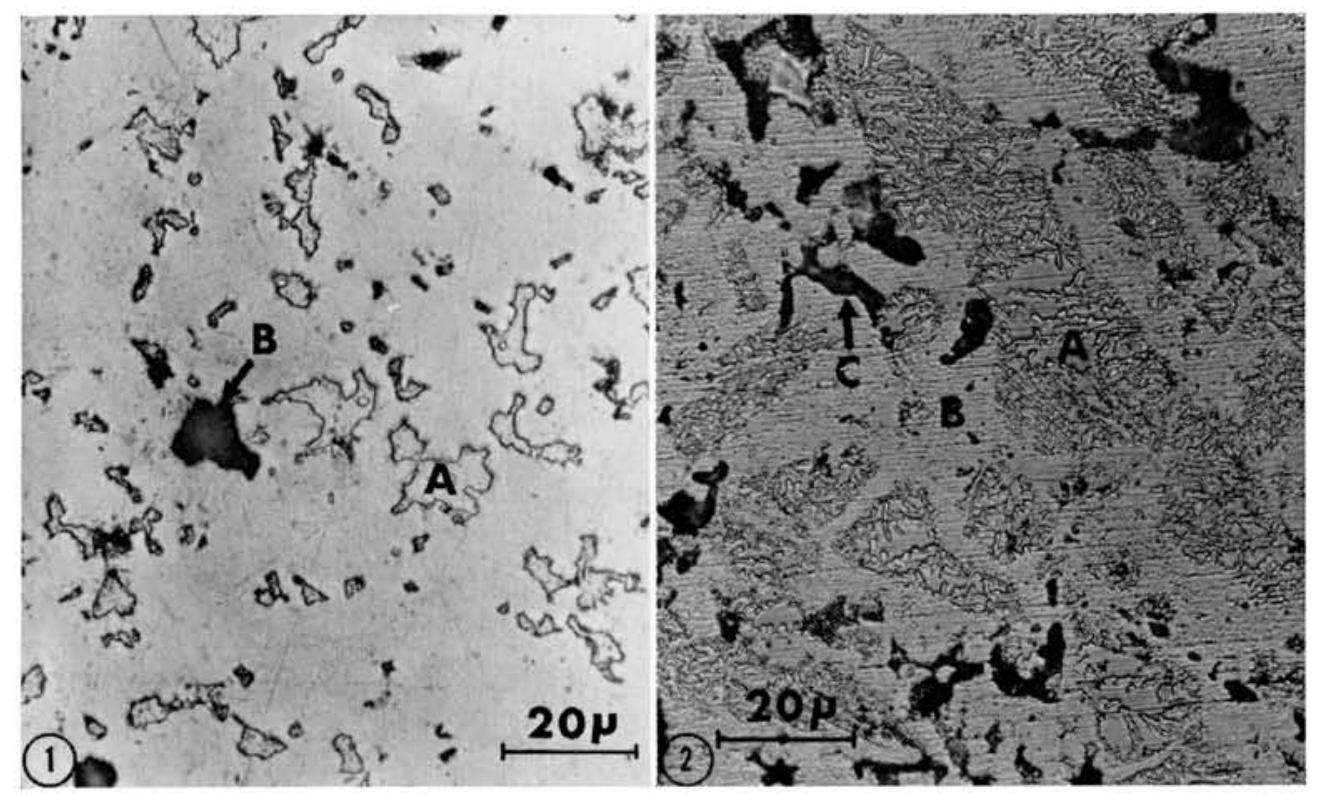

Fig. 1.-Amalgam as polished. $A, \gamma_{2}$ area; $B$, void

FIG. 2.-Amalgam etched with ferric chloride etch. $A$, unreacted original particle; $B, \gamma_{1} ; C$, $\gamma_{2}$ or void 


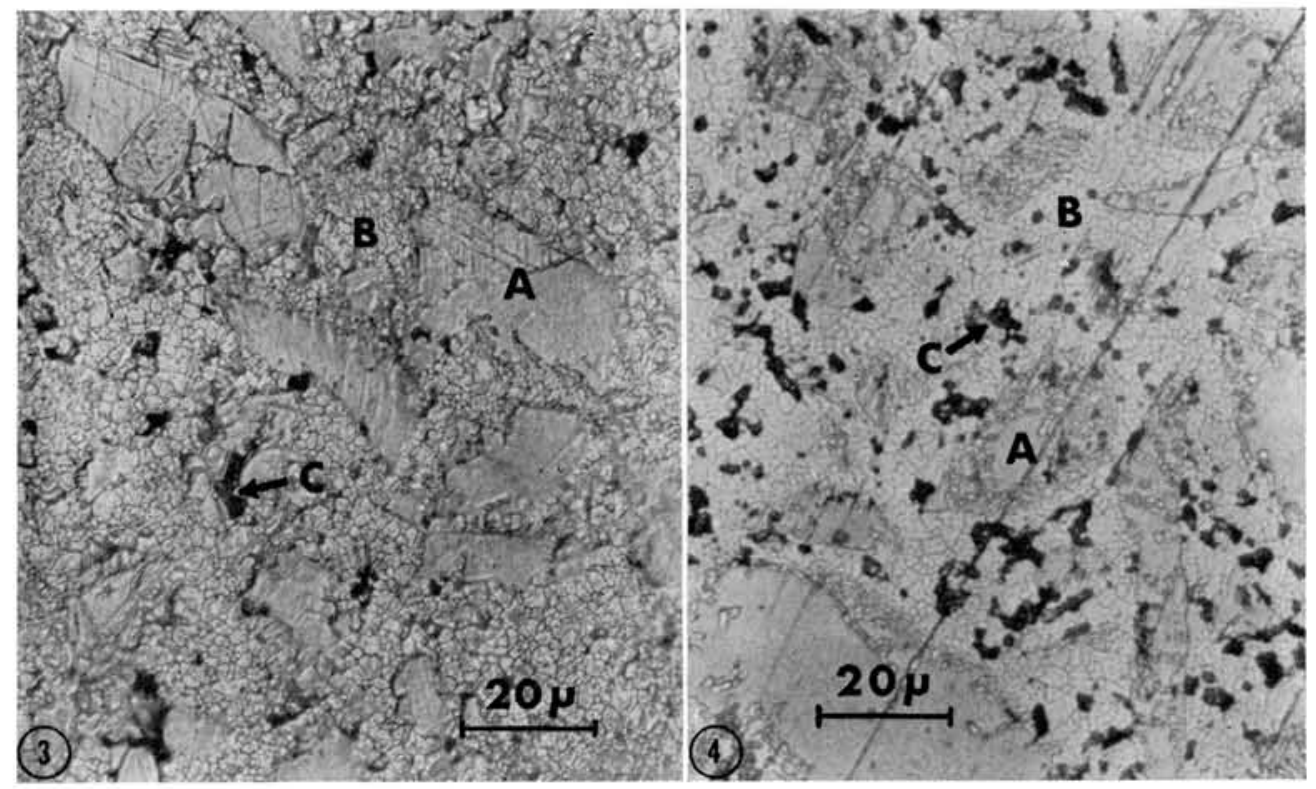

FIG. 3.-Amalgam etched with cyanide etch. $A$, unreacted original particle; $B, \gamma_{1} ; C$, $\gamma_{2}$ or void FIG. 4.-Amalgam etched with nitric acid etch. $A$, unreacted original particle; $B, \gamma_{1} ; C, \gamma_{2}$ or void

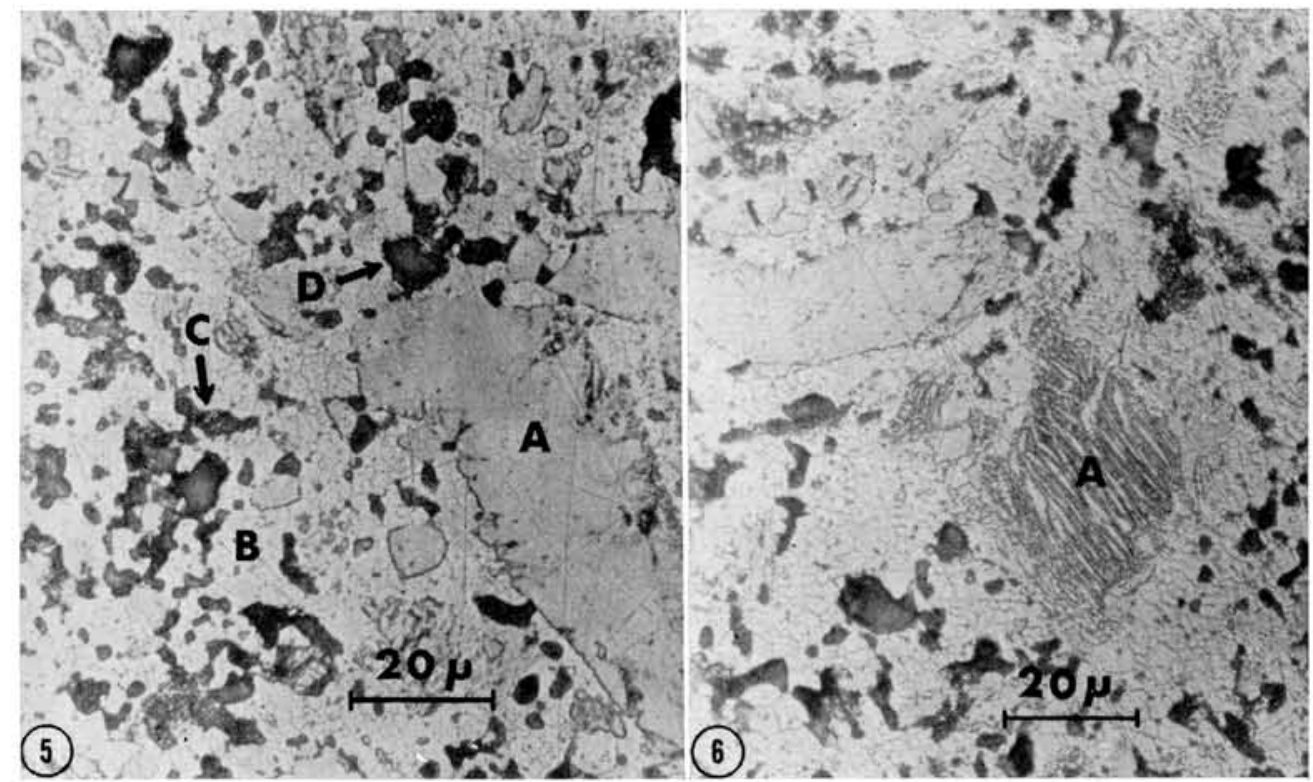

FIG. 5.-Amalgam etched with iodine etch. $A$, unreacted original particle; $B, \gamma_{1} ; C, \gamma_{2} ; D$, void

Fig. 6.-Amalgam etched with iodine etch showing Widmanstatten structure $(A)$ 
For examination of alloy particles, the iodine portion (Solution B) of the etch is sufficient to describe the grain boundaries and Widmanstatten structure. Figure $7, A$, shows the structure obtained after $30 \mathrm{sec}-$ onds of etching. Figure $7, B$, shows the results after 2 minutes of etching. The Widmanstatten structure is destroyed, and the grain boundaries are revealed.
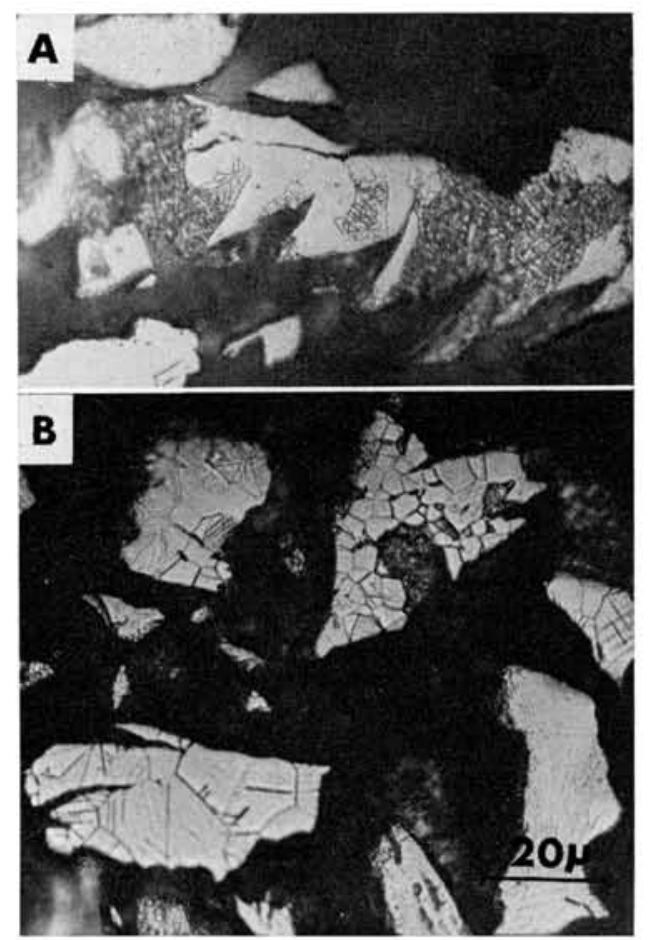

FIg. 7.-Alloy particles etched with Solution B of iodine etch. $A$, etched for 30 seconds, showing Widmanstatten structure; $B$, etched for 2 minutes, showing grain boundaries.

Spherical particles, when etched, showed a dendritic structure. The amalgam showed a uniform structure, with the phases quite easily identifiable (Fig. 8).

Small, light gray-blue areas were dispersed throughout the particles of certain of the alloys. The areas were also present in aged amalgam, along with similar, but larger and darker, areas. These small areas are resistant to the etches used, staining but not etching. Often they appear in a grouping which would suggest a eutectic or lowest melting phase separating out. These areas were analyzed with the electron probe, and photomicrographs are shown in the following section.

An amalgam filling which had been in use for about five years was examined. The amalgam had more voids than are normally present, especially where it approximated the tooth structure. Essentially, the microstructure was the same as that of the other amalgams (Fig. 9). Some $\mathrm{Ag}_{2} \mathrm{Hg}_{3}$ grains appeared quite large and were more resistant to etching than the smaller grains. To some extent, the light gray-blue areas were present, but a greater amount of relatively large dark gray-blue areas were present, which were also etch-resistant. These light and dark areas were also selected for probe analysis.

Microprobe study.-A characteristic curve for dental amalgam is shown in Figure 10. Tracings of $\mathrm{Ag}, \mathrm{Sn}, \mathrm{Hg}$, and $\mathrm{Cu}$ can be seen. $\mathrm{Zn}$ is determined separately when needed, since interference with Sn occurs in a normal determination. These curves were run strictly on a qualitative basis, and no attempt was made to keep track of the variables involved in making a quantitative determination. Therefore, the heights of the tracings for the various elements do not give a direct indication of the percentage of each element present.

The dark gray areas in the aged amalgam sample consisted mostly of Sn. On the portion of the sample where the gray areas were not present, a normal reading was produced (Fig. 11). Since these two curves were run on the same sample at the same amplitude, the heights of the tracings for angles which correspond can be compared. Therefore, the difference in height for the tin tracings indicates a larger percentage of tin present in Area A than in Area B.

When the light and dark areas occurring together were examined, a high concentration of $\mathrm{Sn}$ and $\mathrm{Cu}$ was found (Fig. 12). Further investigation of light gray areas in a non-zinc amalgam revealed again a $\mathrm{Cu}-\mathrm{Sn}$ concentration (Fig. 13). Possibly this concentration could be the $\mathrm{Cu}_{3} \mathrm{Sn}$ phase proposed by Crowell.

Researchers have considered the $\gamma_{2}$ areas to be composed solely of $\mathrm{Sn}$ and $\mathrm{Hg}$. How- 


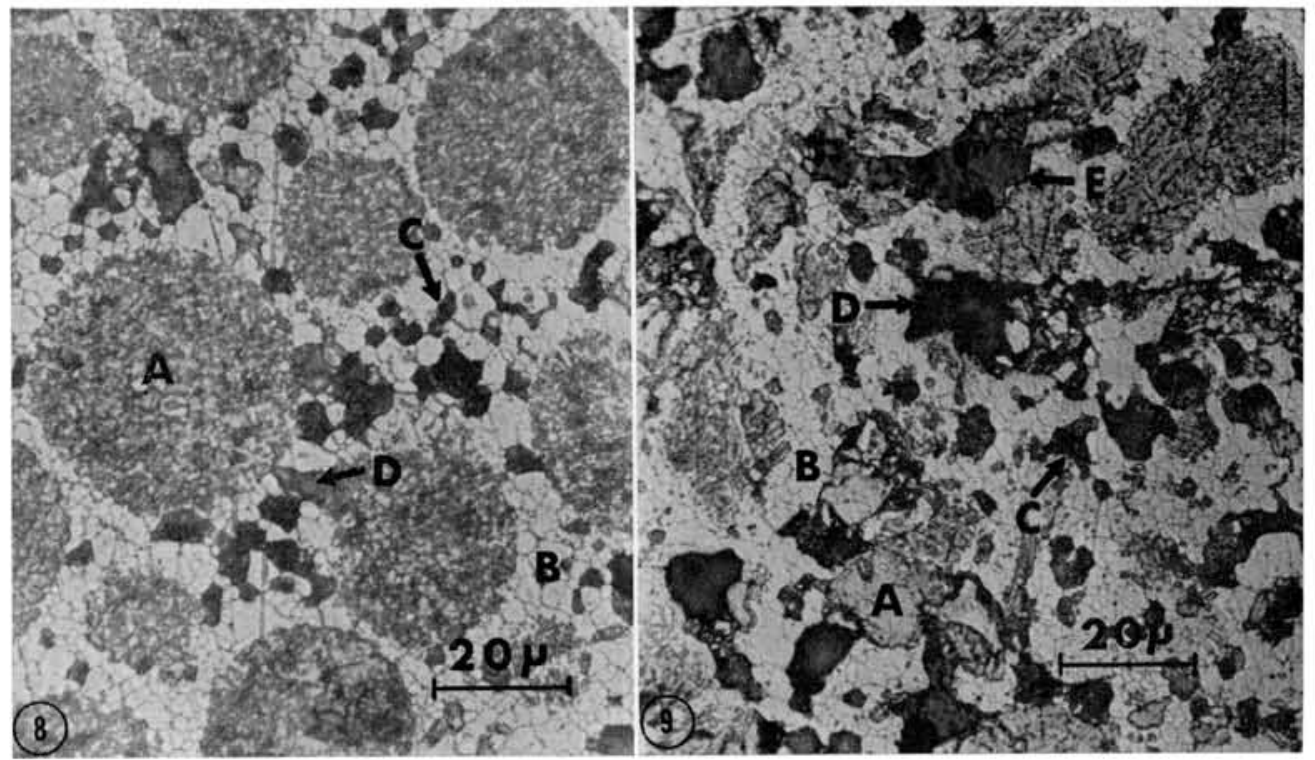

FIG. 8.-Amalgam made from spherical alloy particles, etched with iodine etch. $A$, original particle; $B, \gamma_{1} ; C, \gamma_{2} ; D$, void.

FIG. 9.-Aged amalgam sample, etched with iodine etch. $A$, unreacted original particle; $B, \gamma_{1} ; C, \gamma_{2}$; $D$, void; $E$, dark gray area.

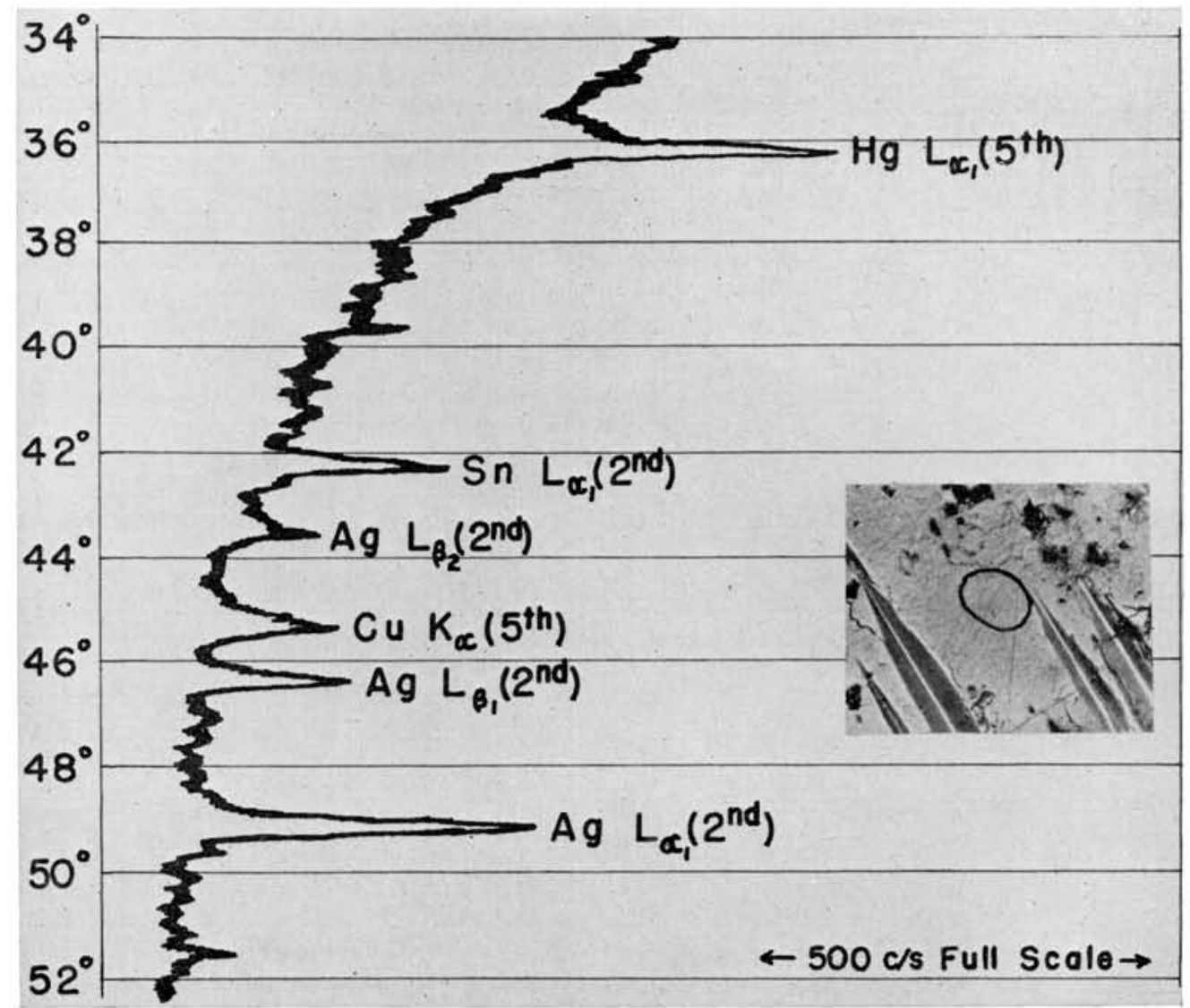

FIG. 10.-Characteristic curve and photograph of area traced 


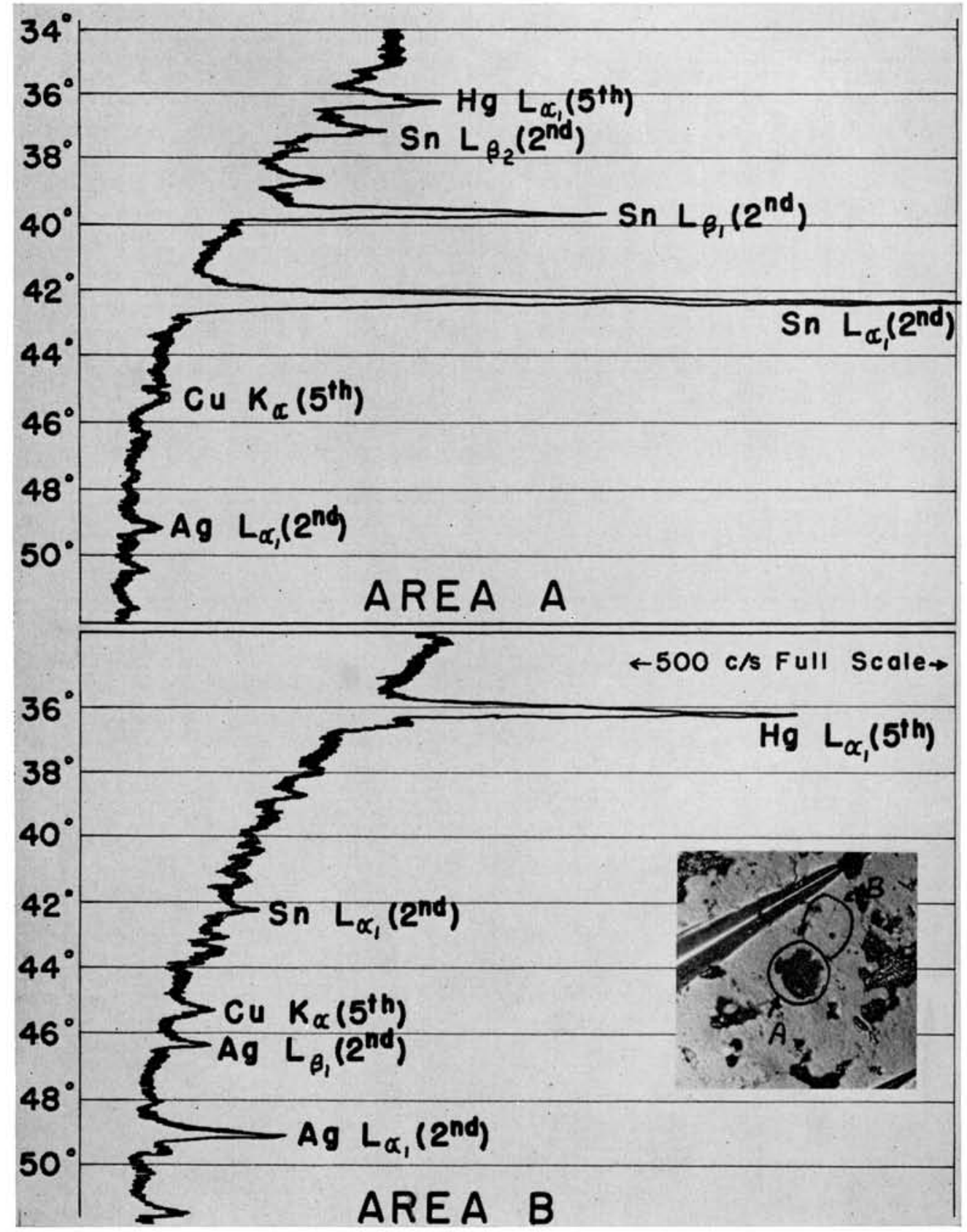

Fig. 11.-Curves of dark gray areas $(A)$ and adjacent area $(B)$ in aged amalgam, and corresponding photograph. 


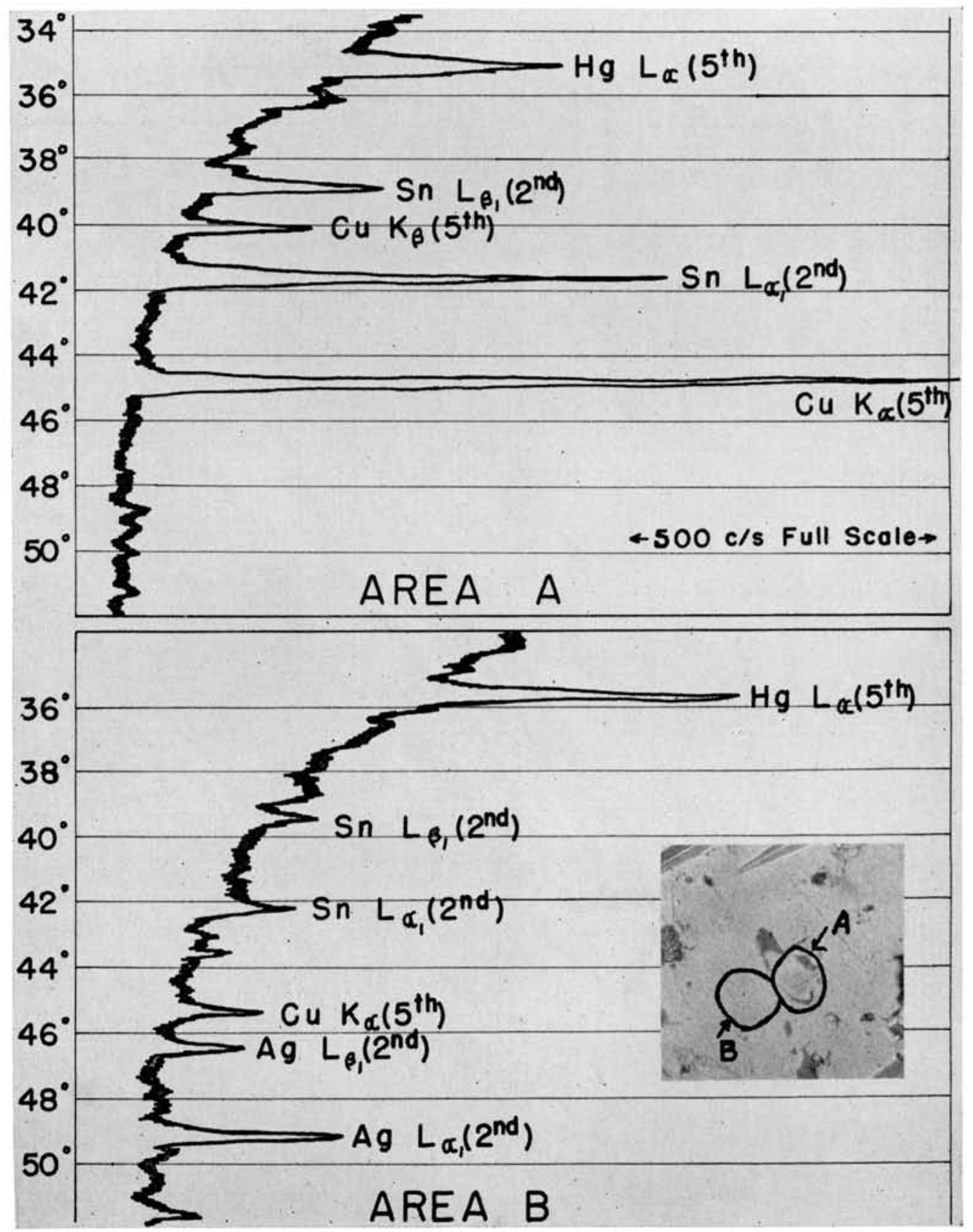

Fig. 12.-Curves of light and dark areas combined $(A)$ and adjacent area $(B)$ in aged amalgam, and corresponding photographs. 


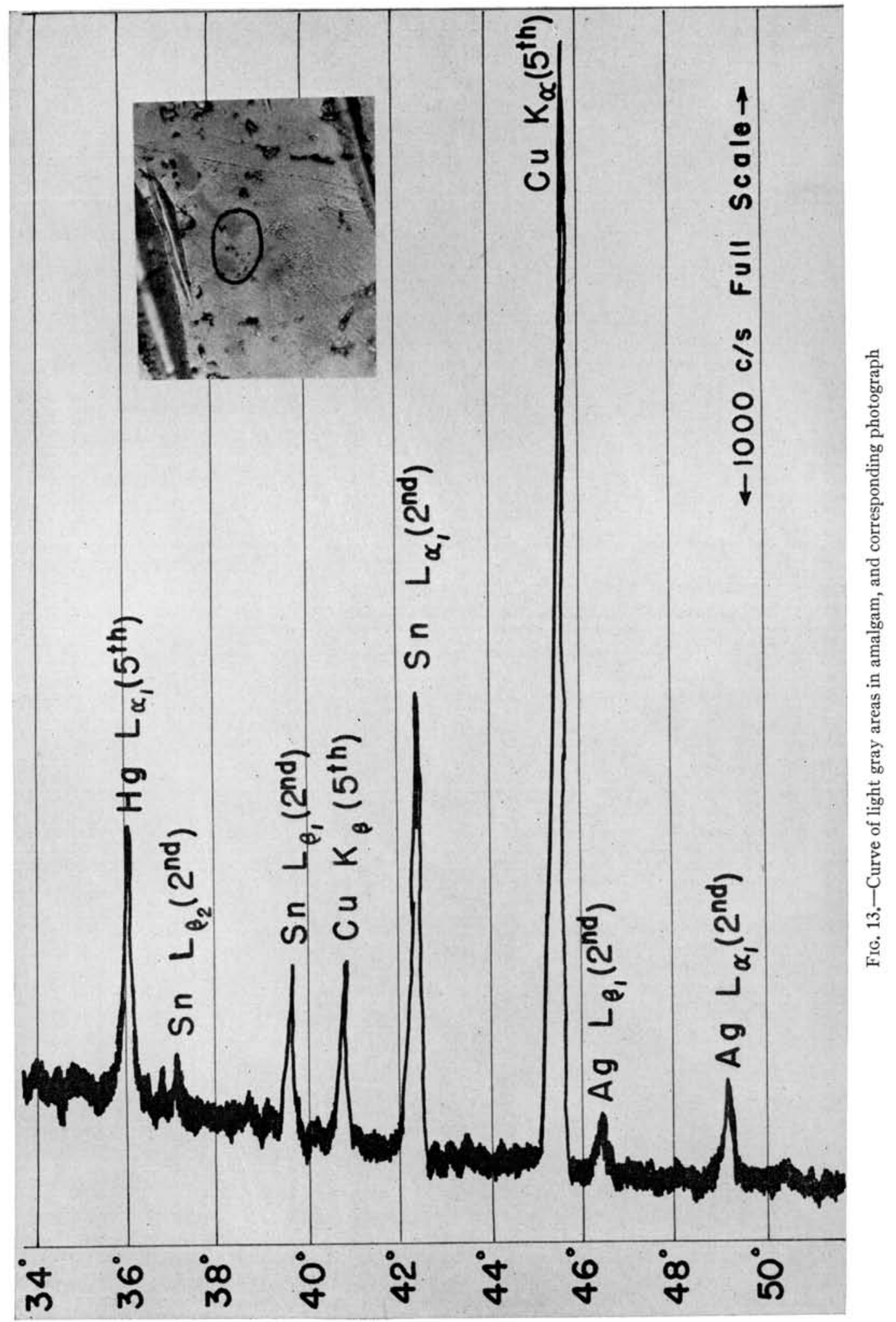



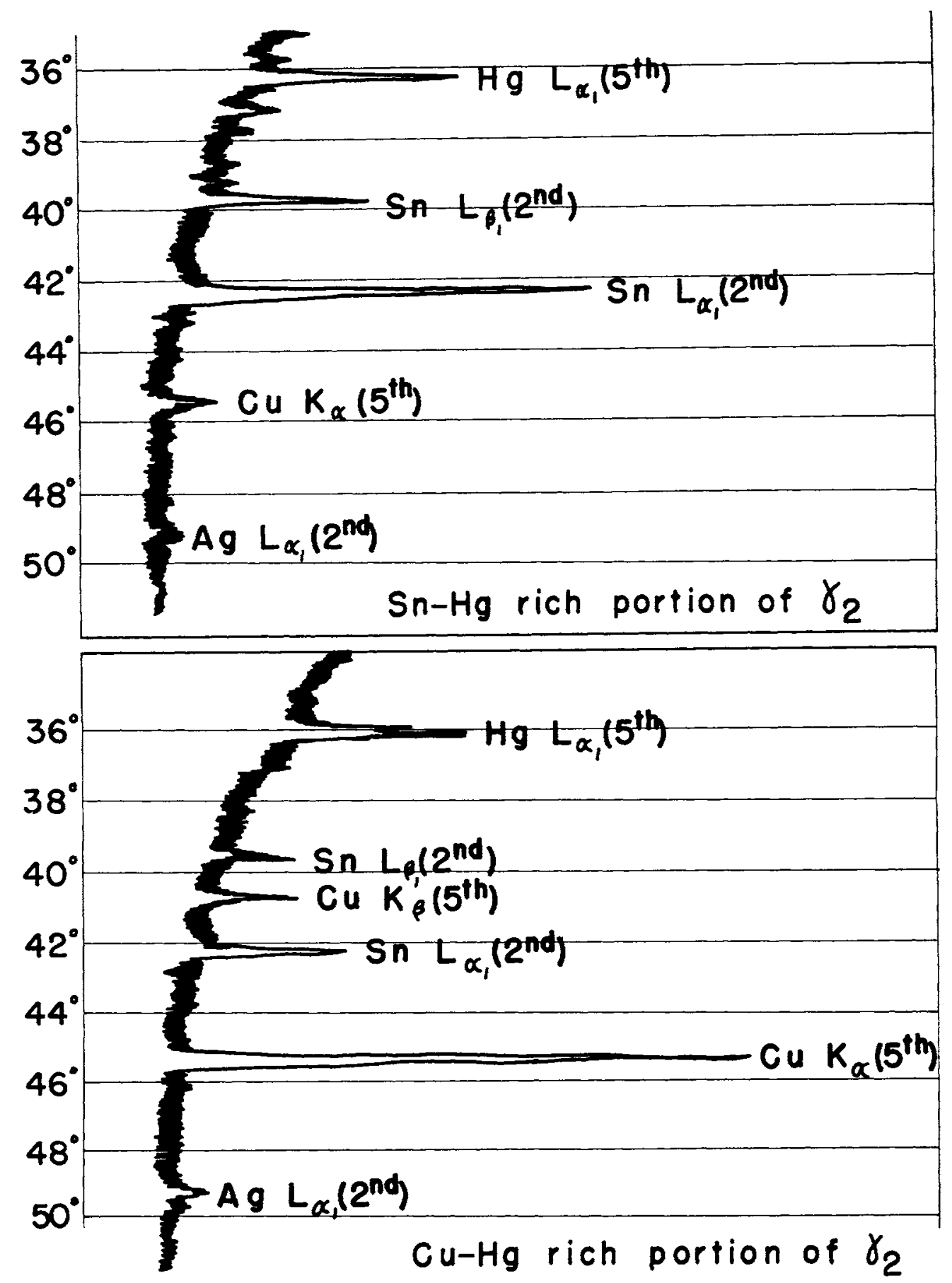

FIG. 14.-Curves for $\gamma_{2}$ area showing $\mathrm{Cu}$-rich portion and Sn-rich portion 
ever, when analyzed, separate areas of either $\mathrm{Cu}-\mathrm{Hg}$ or $\mathrm{Sn}-\mathrm{Hg}$ were found (Fig. 14). When scans were run separately for each element, nowhere within a $\gamma_{2}$ area were $\mathrm{Cu}$ and $\mathrm{Sn}$ found together; therefore, these areas must be a mixture of $\mathrm{Cu}-\mathrm{Hg}$ and $\mathrm{Sn}-\mathrm{Hg}$ phases. This finding could explain the variation in etching of these areas, depending on whether the $\mathrm{Cu}-\mathrm{Hg}$ or $\mathrm{Sn}-\mathrm{Hg}$ phase predominates.

\section{Summary}

By the method outlined, amalgam can be polished with the retention of all the phases. Furthermore, the recommended iodine etch will show, with good contrast, all the phases.

The gray etch-resistant areas have been shown by probe results to consist of $\mathrm{Cu}$ and $\mathrm{Sn}$. Also the dark gray areas appearing in the aged amalgam are predominantly Sn. The $\gamma_{2}$ areas, assumed to be a $\mathrm{Sn}-\mathrm{Hg}$ phase, have been shown to be a mixture of $\mathrm{Sn}-\mathrm{Hg}$ and $\mathrm{Cu}-\mathrm{Hg}$ phases.

\section{References}

1. Sснмітт, G. Über das Gefüge von Amalgamfüllungen verschiedener Zusamensetzung und Herstellung, $D t s c h$. zahnärzll. Z., 15:736-51, 1960.

2. WING, G. The Metallography of Dental Amalgam, pp. 1-201, Unpublished D.D.S. thesis, University of Sydney, Sydney, Australia, 1961.

3. CROWELL, W. S. The Metallography of Dental Amalgam Alloys, J. dent. Res., 33:592-95, 1954.

4. SmtTh, D. L., Ferguson, G. W., and Schoonover, I. C. Microstructure of Dental Amalgam, J. Amer. dent. Ass., 47:305-11, 1953.

5. Ryge, G., FaIrhurst, C. W., and Fischer, C. H. The Present Knowledge of the Mechanism of the Setting of Dental Amalgam, Int. dent. J., 11:181-95, 1961.

6. Fatrhurst, C. W. and Ryge, G., X-Ray Diffraction Investigation of the $\mathrm{Sn}-\mathrm{Hg}$ Phase in Dental Amalgam, Advances in X-Ray Analysis, 5:64-70, 1962.

7. Symposium on Methods of Metallographic Specimen Preparation, ASTM Special Technical Publication No. $285,1960$.

8. KeHL, G. L. Principles of Metallographic Laboratory Practice, pp. 1-79. 3d ed. New York: McGraw-Hill, 1949.

9. Berglund, T. Metallographer's Handbook of Elching. Translated by W. H. DEarden. London: Pitman and Sons, Ltd., 1931.

10. Metals Handbook. Cleveland, Ohio: American Society for Metals.

11. BrRks, L. S. Electron Probe Microanalysis, pp. 1-253. New York \& London: Interscience Publishers, 1963. 